\title{
Lernen autonome Fahrzeuge?
}

\author{
Walther Wachenfeld, Hermann Winner
}

\section{Inhaltsverzeichnis}

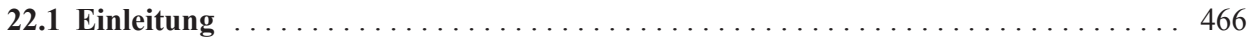

22.2 Fahrzeug, Umwelt und lernender Fahrer $\ldots \ldots \ldots \ldots \ldots \ldots \ldots \ldots \ldots \ldots \ldots$

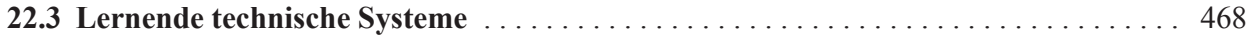

22.3.1 Verschiedene Verfahren des Maschinellen Lernens . . . . . . . . . . . . . . . . . . . 469

22.3.2 Beispiele ................................... 471

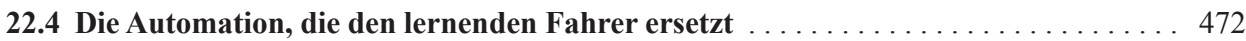

22.4.1 Sicherheitsrelevante Systeme . . . . . . . . . . . . . . . . . . 473

22.4.2 Herausforderungen und Lösungsansätze in den verschiedenen Phasen

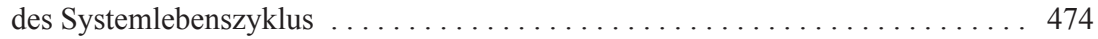

22.4.3 Maße sicheren Fahrens . . . . . . . . . . . . . . . . . . . . . . . . . . . 479

22.5 Die Automation als Teil eines lernenden Kollektivs $\ldots \ldots \ldots \ldots \ldots \ldots \ldots \ldots . \ldots$

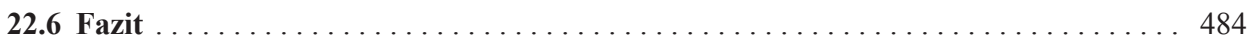

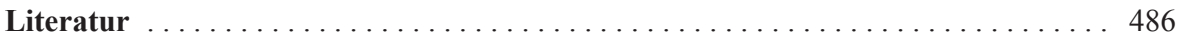

W. Wachenfeld $(\square)$

Technische Universität Darmstadt, Fachgebiet Fahrzeugtechnik - FZD, Deutschland wachenfeld@fzd.tu-darmstadt.de

H. Winner

Technische Universität Darmstadt, Fachgebiet Fahrzeugtechnik - FZD, Deutschland winner@fzd.tu-darmstadt.de 


\subsection{Einleitung}

Mit dem autonomen Fahren wird ein technisches System den Menschen als Fahrer des Automobils ersetzen. Aktuell arbeiten die Automobilindustrie, Universitäten, aber auch große IT-Unternehmen an der Implementierung von Funktionen, die dem technischen System erlauben, die Fahrzeugführung umzusetzen. Dabei stehen die Aufgaben der Perzeption, Kognition, Verhaltensentscheidung und die Verhaltensausführung, die auch der Mensch vollbringt, im Fokus. Zusätzlich besitzt der Mensch weitere Fähigkeiten, die nicht direkt mit dem Führen eines Fahrzeugs in Verbindung stehen. Beispielsweise verändert das Lernen des Menschen direkt die Leistungsfähigkeit, mit der er Aufgaben bewältigt. Diese Fähigkeit des Menschen im System Fahrer - Fahrzeug - Umwelt eröffnet die Frage, ob für das Ersetzen des Menschen das technische System ebenfalls Lernfunktionen aufweisen wird. In den verschiedensten Bereichen, getrieben vor allem durch die Informatik, existieren lernende und gelernte Systeme unterschiedlichster Form, die es mit herkömmlichen analytischen Systemen und deren Leistungsfähigkeit aufnehmen können. Das Besondere der Fahrzeugautomatisierung ist jedoch zum einen deren Sicherheitsrelevanz, zum anderen unterscheidet sich das Produkt Automobil zusätzlich durch seine Systemlebenszyklen von anderen Gütern aus der IT-Industrie. Diese beiden Besonderheiten mit ihren Herausforderungen und Lösungsansätzen werden diskutiert. Zusätzlich wird auf kollektives Lernen im Zusammenhang des autonomen Fahrens eingegangen, denn besonders der direkte Austausch bzw. die Kopie von Gelerntem stellt einen der Vorteile des maschinellen Lernens gegenüber dem menschlichen Lernprozess dar.

Das Nachbilden des menschlichen Lernens durch Maschinelles Lernen beschäftigt eine ganze Forschergemeinde, denn sowohl über die Prozesse des menschlichen Lernens als auch für Methoden des Maschinellen Lernens wird daraus ein weiterführendes Verständnis als Ergebnis erwartet. Mit dem Bewusstsein über den aktuell vorhandenen Unterschied zwischen beiden Formen des Lernens wird in diesem Kapitel Maschinelles Lernen als Algorithmus verstanden, der durch einen Menschen erzeugt wird. Die Ausführung der Software folgt diesen Algorithmen wie jede andere Software auch. Ziel dieses Beitrags ist es nicht, den lernenden Menschen mit einem lernenden Fahrroboter zu vergleichen. Vielmehr wird diskutiert, warum, ob und mit welchen Herausforderungen und Lösungsansätzen Maschinelles Lernen in seiner aktuellen Form im autonomen Fahrzeug denkbar ist. Dabei stellt dieses Kapitel im Besonderen die Sicht der Fahrzeugtechnik auf diese Frage dar und basiert für den Bereich des Maschinellen Lernens auf Erfahrungen aus der Literatur.

\subsection{Fahrzeug, Umwelt und lernender Fahrer}

Führt der Fahrer ein Fahrzeug in einer beliebigen Umwelt, handelt es sich dabei um eine zielgerichtete Tätigkeit. Nach Rasmussen [1, 2] zeigt der Mensch dabei ein Verhalten, das sich in drei Bereiche aufteilen lässt. Er handelt fertigkeits-, regel- und wissensbasiert (s. Abb. 21.6 in Kap. 21). Fertigkeitsbasiertes Verhalten wird von Rasmussen [1] als Reiz- 
Reaktions-Automatismus beschrieben, den der Mensch in alltäglichen Routinesituationen ohne intensive Inanspruchnahme von kognitiven Fähigkeiten bewältigt.

Kognitiv anspruchsvoller gestaltet sich das regelbasierte Verhalten. Zusätzlich zur Wahrnehmung und zur motorischen Aktion ist für das regelbasierte Verhalten eine assoziative Zuordnung notwendig. Hierbei ordnet der Mensch die erkannte Situation einer ihm bekannten Regel zu und wählt basierend darauf aus einem Repertoire an Verhaltensregeln. Diese Regeln hat der Mensch zielgerichtet gelernt oder sich von vergangenen Situationen und Handlungen gemerkt (,gespeichert“). In diesem Zusammenhang besitzt der Mensch die Fähigkeit, ähnliche Situationen zu identifizieren und somit gelernte Regeln auf ähnliche Situationen zu übertragen.

Treten Situationen auf, die für den Menschen neu sind und für die keine trainierten Handlungen vorliegen, agiert der Mensch wissensbasiert. Dazu versucht der Mensch, basierend auf seinem trainierten Wissen, die Handlungsalternativen, die zur Auswahl stehen, zu generieren und zu bewerten. Die subjektiv optimale Alternative wird ausgewählt und ausgeführt.

Rasmussens [1] Verständnis von zielgerichtetem Handeln macht deutlich, was mit dem lernenden Fahrer gemeint ist. Zu Beginn der Fahrer-,,Karriere“ wird in der Theorieausbildung der Fahrschule das Grundlagenwissen über den Straßenverkehr aufgebaut und abgeprüft. Dabei setzt dieses Wissen auf dem bereits erworbenen Wissen durch das Leben in der Gesellschaft auf. Zusätzlich wird regelbasiertes Verhalten durch Theorie- und Praxisunterricht trainiert. Mit abgelegter Führerscheinprüfung darf der Mensch nun ohne weitere Unterstützung am öffentlichen Straßenverkehr teilnehmen (Ausnahme: begleitendes Fahren beim Führerschein mit 17). Jedoch hat der Mensch zu diesem Zeitpunkt weder alle Regeln erlernt noch das Wissen erarbeitet, das für das zukünftige Leben im öffentlichen Straßenverkehr benötigt wird. Mit jeder Erfahrung, die Menschen sammeln, verschiebt sich das Verhalten von wissensbasiert zu regelbasiert und von regelbasiert zu fertigkeitsbasiert. Das Training ermöglicht somit eine Effizienzsteigerung des menschlichen Verhaltens [1].

Zieht man Zahlen für verunglückte Pkw-Fahrer pro einer Million Kilometer Verkehrsleistung heran, dann sinkt nach Oswald und Williams [3, 4] das Risiko mit dem fortschreitenden menschlichen Alter, bis es ab 40-50 wieder zu steigen beginnt. Nach Burgard [5] sind dafür neben charakterlichen Kompetenzen (Persönlichkeit) sowie psychischen und physischen Voraussetzungen auch die Erfahrungen verantwortlich, die aus dem Alter folgen. Wird das Verarbeiten der Erfahrungen als Lernprozess gesehen, trägt die Fähigkeit des Lernens zu einer Verbesserung der Fahrkompetenz bei [6].

Würde der Straßenverkehr klaren Regeln folgen, die dem Menschen bekannt sind, so müsste er das oben beschriebene Verhalten nicht zeigen. Es handelt sich beim Straßenverkehr jedoch um ein offenes System, bestehend aus statischen und dynamischen Objekten sowie einer Vielzahl von Umfeldeinflüssen, wie beispielsweise Helligkeit oder Niederschlag. Wenn auch in geringerem Umfang, verglichen mit Fahranfänger/innen, treten dadurch für erfahrene Teilnehmer des Straßenverkehrs weiterhin unbekannte Situationen auf, die es zu bewältigen gilt. Weil der Mensch eben dieses wissens-, regel- und fertigkeits- 
basierte Verhalten zeigt, ist der aktuelle Straßenverkehr mit der vorhandenen Effizienz, Genauigkeit und Sicherheit möglich.

Zusätzlich führt dieses Verhalten zu einem individuellen Fahrverhalten. In ein und derselben Situation handeln Fahrer unterschiedlich und haben verschiedene Vorlieben für die Wahl des Abstands, der Geschwindigkeit und der Beschleunigungen.

Eben diese Fähigkeiten, die das fertigkeits-, regel- und wissensbasierte Verhalten ermöglichen, werden mit der Automatisierung aus der Fahrzeugführung genommen und durch entsprechende Fähigkeiten des Fahrroboters ersetzt.

\subsection{Lernende technische Systeme}

Der Begriff des Maschinellen Lernens steht für ein Forschungsgebiet, das sich mit Methoden für die Erstellung von Algorithmen beschäftigt. Eine besondere Eigenschaft dieser Algorithmen ist die automatische, auf Erfahrungen basierende Verbesserung des technischen Systems. Die automatische Verbesserung folgt dabei den Regeln und Maßen, die der menschliche Entwickler zuvor definiert hat. Kontrovers diskutierte Vorstellungen von vollkommen frei und kreativ handelnden Maschinen, wenn es diese überhaupt geben wird, sind nicht im Fokus dieser Betrachtung. Für den Einsatz des Maschinellen Lernens wird eine klar definierte Aufgabe benötigt, für die eine Bewertungsmetrik sowie (Trainings-)Daten zur Verfügung stehen.

Ein oft verwendetes Zitat für die Definition von Maschinellem Lernen stammt von Mitchell:

A computer program is said to learn from experience $\mathrm{E}$ with respect to some class of tasks

$\mathrm{T}$ and performance measure $\mathrm{P}$, if its performance at tasks in $\mathrm{T}$, as measured by $\mathrm{P}$, improves with experience E. [7]

Nach Mitchell [7] hat sich Maschinelles Lernen im Einsatz besonders dann bewährt, wenn

1. eine große Anzahl von Daten in einer Datenbank existiert und diese möglicherweise implizit Informationen enthält, die automatisiert extrahiert werden können,

2. Menschen über einen gewissen Bereich nur ein schlechtes Verständnis besitzen und somit das Wissen für effektive Algorithmen fehlt oder

3. Aufgaben eine dynamische Anpassung an sich ändernde Umgebungsbedingungen erfordern.

Wie diese Definition und die Einsatzbereiche zum autonomen Fahren passen, wird in Abschn. 22.4 diskutiert. Zunächst wird jedoch ein Einblick in Verfahren des Maschinellen Lernens gegeben. Mithilfe von Beispielen aus verschiedenen Bereichen wird die Breite der Einsatzmöglichkeiten demonstriert, bevor auf das autonome Fahren als besondere Aufgabe eingegangen wird. 


\subsubsection{Verschiedene Verfahren des Maschinellen Lernens}

Breiman stellte 2001 Folgendes fest:

In the past fifteen years, the growth in algorithmic modeling applications and methodology has been rapid. It has occurred largely outside statistics in a new community - often called machine learning. [8]

In [9] werden die ersten Arbeiten des Maschinellen Lernens u. a. auf McCulloch und Pitts im Jahr 1948 zurückgeführt, sodass die Vielzahl der vorhandenen Lernverfahren eine detaillierte Beschreibung aller Verfahren nicht ermöglicht. Deshalb werden nachfolgend Kategorien von Lernproblemen, nach denen die Verfahren zumeist eingeteilt werden $[10,11,12]$, beschrieben.

\section{Supervised Learning}

Supervised Learning, überwachtes Lernen oder Lernen mit Lehrer zeichnet sich durch bereits bewertete Trainingsdaten (labeled data) aus. Die Trainingsdaten, also die Erfahrungen, auf denen das Maschinelle Lernen aufbaut, enthalten Eingangs- und Ausgangsgrößen eines Lernproblems. Beispielsweise würden bei der Klassifikation, ob bei einem Unfall Airbags auslösen oder nicht, Trainingsdaten auf der einen Seite aus Beschleunigungswerten und auf der anderen Seite aus zugehörigen Bewertungen (auslösen oder nicht auslösen) bestehen. Diese Bewertungen müssen dabei beispielsweise durch einen Experten/Lehrer oder eine Beobachtung über der Zeit erstellt werden. Das Lernverfahren nutzt nun diese Erfahrungswerte, um für neu beobachtete Eingangswerte die Ausgangsgröße zu bestimmen. Für das Übertragen von Erfahrungswerten auf neue Eingangswerte wird nach [10] zwischen dem faulen (lazy- bzw. memory-based learning) und dem eifrigen Lernen (eager bzw. model-based learning) unterschieden.

Beim faulen Lernen werden die Trainingsdaten während des Lernens abgespeichert und ein Ähnlichkeitsmaß definiert. Dieses Ähnlichkeitsmaß kann unterschiedlich komplex sein und reicht vom einfachen euklidischen Abstand bis zu komplexen Abständen beim CaseBased-Reasoning. Wenn der Ausgangswert für neue Eingangswerte gesucht wird, werden die dem neuen Fall ähnlichsten Trainingsdaten bestimmt und daraus der Ausgangswert abgeleitet. Dieses Vorgehen entspricht dem transduktiven Schlussfolgern [13]. Im Gegensatz dazu wird beim eifrigen Lernen, basierend auf den Trainingsdaten, während der Trainingsphase ein globales Modell erstellt (Induktion). Ausgangswerte für neue Fälle werden per Deduktion aus dem Modell geschlossen.

\section{Unsupervised Learning}

Für das Unsupervised Learning, unüberwachtes Lernen oder Lernen ohne Lehrer stehen ebenfalls Trainingsdaten zur Verfügung, jedoch in diesem Fall ohne Bewertung bzw. Ausgangsgröße (unlabeled data). Bei diesem Lernverfahren ist das Ziel, eine Struktur in den Daten zu finden bzw. diese anhand der Struktur zu klassifizieren. Die Trainingsdaten wer- 
den dabei genutzt, um diese Strukturen aufzudecken und neu beobachtete Eingangswerte basierend darauf einzuteilen.

\section{Reinforcement Learning}

Das Reinforcement Learning oder Lernen durch Verstärkung unterscheidet sich von beiden vorherigen Verfahren, denn zu Beginn liegen keine oder wenige Trainingsdaten vor. Die

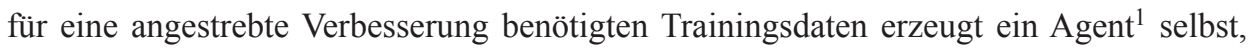
indem nach einem festgelegten Schema die zu optimierende Aufgabe ausgeführt wird. Eine Bewertung der Ausführung der Aufgabe fließt in den Lernprozess zurück und bildet so einen Trainingsdatensatz aus Ein- und Ausgangsgrößen, die für weitere Optimierungsschritte genutzt werden. Die Ansätze des Reinforcement Learnings sind dem sogenannten Innovationsdilemma ausgesetzt, denn „Exploration“ und „Exploitation“ widersprechen einander, was March folgendermaßen beschreibt:

Exploration includes things captured by terms such as search, variation, risk taking, experimentation, play, flexibility, discovery, innovation. Exploitation includes such things as refinement, choice, production, efficiency, selection, implementation, execution. [15]

Dem Lernproblem entsprechend ist ein Gleichgewicht für beides zu finden, denn auf der einen Seite ist in einem teils unbekannten Suchraum eine optimale Ausführung der Aufgabe gesucht und auf der anderen Seite wird diese Suche durch Rahmenbedingungen wie Kosten, Sicherheit und Zeit begrenzt.

Neben der Frage, ob und in welcher Form die Trainingsdaten vorhanden sind, kann das Lernproblem auch anhand des Einsatzes der Trainingsdaten unterschieden werden.

\section{Batch Learning}

Beim Batch oder Offline Learning wird zu einem Zeitpunkt ein Satz von Trainingsdaten verwendet, um die Lernmethode anzuwenden. Erstellt die Lernmethode beispielsweise ein Modell, wird dieses Modell durch weitere Erfahrungen, die während des Einsatzes gesammelt werden, nicht aktualisiert.

\section{Online Learning}

Das Online Learning zeichnet sich durch einen iterativen Prozess aus, bei dem neue Erfahrungen in den Lernprozess mit einfließen. Das Ziel ist, die Bewältigung der Aufgabe kontinuierlich zu optimieren und dabei Erfahrungen aus dem Einsatz zu berücksichtigen. Das hat ein Systemverhalten zur Folge, das sich über den Erfahrungen ändert. Da die Erfahrungen in einer zeitlichen Abfolge gesammelt werden, ändert sich das Systemverhalten demzufolge auch über der Zeit.

1 Russell definiert: "An agent is anything that can be viewed as perceiving its environment through sensors and acting upon that environment through effectors." [14] 
Diese unterschiedlichen Arten von Lernproblemen erfordern den Einsatz verschiedener Methoden des Maschinellen Lernens [12]. Diese reichen von Entscheidungsbäumen, künstlichen Neuronalen Netzen und Genetischen Algorithmen über Stützvektorenmethode, Instanzenbasiertes Lernen, Hidden-Markov-Modelle, Wert-Iteration bis hin zum Q-Lernen usw. Diesen Methoden ist gemein, dass die Güte, mit der ein Lernproblem bearbeitet wird, von drei grundlegenden Eigenschaften abhängt. Zum einen wird die Repräsentativität der Trainingsdaten gefordert, denn nur wenn auch für den Betrieb relevante Daten (Erfahrungen) in ausreichender Anzahl für das Lernen eingesetzt werden, sind die Methoden in der Lage, das Lernproblem optimal zu lösen. Zum anderen gilt Gleiches für die Qualität der Trainingsdaten, sodass gerade für real gemessene Größen die Behandlung von verrauschten, fehler- oder lückenhaften Daten notwendig ist. Neben den Testdaten stellt die Bewertung der Performanz $(\mathrm{P})$ eine weitere Herausforderung dar. Nur wenn die Bewertung für den gesamten Einsatzbereich mit realen Daten valide ist, wird auch die Methode das Lernproblem korrekt bearbeiten.

\subsubsection{Beispiele}

Folgende Beispiele dienen als kleine Stichprobe für Bereiche, die mit Verfahren des Maschinellen Lernens adressiert werden können.

\section{Airbagauslösung [16, 17]}

Das Lernproblem besteht aus der Zuordnung von Sensorwerten zu dem Befehl der Auslösung eines Fahrzeugairbags. Dafür wird ein Klassifikator gelernt, der Unfälle entweder der Klasse ,auslösen“ oder „nicht auslösen“ zuordnet. In diesem Beispiel hat der Datensatz eines Unfalls 30 Dimensionen und besteht beispielsweise aus Beschleunigungs-, Druckoder Körperschallsensoren an verschiedenen Punkten im Fahrzeug. Für 40 Trainingsdatensätze, bei denen die Sensorwerte für repräsentative Unfälle aufgezeichnet werden, wird zusätzlich bewertet (gelabelt), ob eine Auslösung der Airbags notwendig wäre oder nicht.

\section{Fahrtrajektoriensteuerung mit KNN [18]}

Das Projekt ALVINN (autonomous land vehicle in a neural network) hatte das Ziel, ein Fahrzeug auf der optimalen Fahrtrajektorie innerhalb seines Fahrstreifens zu positionieren. Die Trainingsdaten bestehen aus den Eingangsgrößen, den einzelnen Pixeln eines Kamerabildes und der zugehörigen Ausgangsgröße, dem Lenkwinkel. Aufgezeichnet werden diese während der Fahrt eines menschlichen Fahrers. Gelernt wird ein Künstliches-Neuronales-Netz, an dessen 960 Eingangsknoten die einzelnen Werte der 30 x 32-Pixel des Kamerabildes übergeben werden. Diese Eingangsknoten sind über vier versteckte Knoten mit den 30 Ausgabeknoten verbunden, die jeweils für eine eigene Krümmung stehen. 


\subsection{Die Automation, die den lernenden Fahrer ersetzt}

Für die Automobilindustrie ist Maschinelles Lernen und Künstliche Intelligenz nicht nur bei der Automatisierung der Fahrzeugführung von Interesse, sondern auch in anderen Bereichen wie dem Design, der Produktion oder dem After-Sales-Management [19]. Diese Bereiche werden jedoch für die Betrachtung ausgeschlossen, genauso wie der Bereich des Infotainments. Im Fokus steht die Fahrzeugautomatisierung, die nach heutigem Verständnis aus folgenden Komponenten besteht, um ein Fahrzeug im öffentlichen Straßenverkehr zu bewegen:

1. Perzeption von Umfeld- und Fahrzeugzustandsgrößen,

2. Kognition dieser Größen zu einer Weltrepräsentation,

3. Verhaltensentscheidung basierend auf der Weltrepräsentation und

4. Verhaltensausführung.

Diese Komponenten liefern die Eigenschaften, die nach Mitchell (s. Abschn. 22.3) bereits zu einem erfolgreichen Einsatz von Maschinellem Lernen geführt haben. Die zunehmende Perzeption von Umfeld- sowie Fahrzeugzustandsgrößen liefert eine Vielzahl maschinenlesbarer Informationen. Denn zum einen nimmt die Leistungsfähigkeit der Sensoren und der Signalverarbeitung weiter zu, sodass ein detaillierteres Bild der Welt für die maschinelle Verarbeitung zur Verfügung steht. Zum anderen steigt die Anzahl an Sensoren und damit ausgestatteter Fahrzeuge auf der Roadmap zur Vollautomatisierung. Daraus folgt, dass die Qualität und Quantität an Trainingsdaten für Maschinelles Lernen zunimmt. Die zweite Eigenschaft gilt besonders für manche Bereiche der Kognition und Verhaltensentscheidung, denn für die menschlichen Prozesse, die es zu ersetzen gilt (s. Abschn. 22.2), existieren vorwiegend Theorien. Anhand des von der EU geförderten Human Brain Projects und den damit einhergehenden Forschungsarbeiten ist ersichtlich, dass noch eine Vielzahl von ungelösten Fragestellungen existiert und somit das Wissen für effektive Algorithmen fehlt. Die dritte Eigenschaft liefert der Straßenverkehr. Wie schon in Abschn. 22.2 beschrieben, erfordert die Welt, in der sich das Fahrzeug bewegt, die Anpassung an sich ändernde Umgebungsbedingungen, weshalb eine Implementation von Maschinellem Lernen in der Automation, die sich diesen Änderungen anpassen kann, naheliegt.

Neben diesen drei Motivatoren für den Einsatz des Maschinellen Lernens existieren jedoch auch besondere Herausforderungen, die dem Einsatz entgegenstehen. Das Besondere der für autonomes Fahren notwendigen vier Komponenten (Perzeption, Kognition, Verhaltensentscheidung, Verhaltensausführung) gegenüber anderen Anwendungen des Maschinellen Lernens ist der Eingriff in das reale Verhalten des Fahrzeugs, sodass, unabhängig, an welcher Stelle dieser Kette ein unerwünschtes Verhalten auftritt, sich dieses zu einem Ausfall und einem Unfall weiterentwickeln kann. Um den Einsatz von Verfahren des Maschinellen Lernens in der Fahrzeugautomatisierung zu diskutieren, erfolgt deshalb zunächst die Klassifizierung sicherheitsrelevanter Systeme sowie die Zuordnung der Fahrzeugautomatisierung zu einer dieser Klassen. 


\subsubsection{Sicherheitsrelevante Systeme}

Nach DIN 31000 gilt:

Sicherheit ist eine Sachlage, bei der das Risiko nicht größer als das Grenzrisiko ist. [20]

Inwieweit ein System im Automobil (oder auch generell) diese Sicherheit beeinflusst, kann folgendermaßen eingeteilt werden ([16] erweitert):

1. Sicherheitsunkritisch

Fehler in diesem System führen zu keiner Gefährdung von Personen oder der Umwelt. Eine fehlerhafte Spracherkennung, die oft mit Verfahren des Maschinellen Lernens umgesetzt wird [21], ist beispielsweise sicherheitsunkritisch, wenn diese im Infotainment genutzt wird. Deshalb sind solche Systeme bereits im Einsatz, obwohl zum Teil regelmäßig Fehler auftreten, ohne dass die Sicherheit negativ beeinflusst wird.

2. Sicherheitsrelevant

Ein System wird als sicherheitsrelevant bezeichnet, wenn durch Fehler dieses Systems eine Gefährdung von Personen oder der Umwelt eintreten kann.

c. System zur Entscheidungsunterstützung

Hier können die Vorschläge des Systems durch den Entscheider ausgeführt werden oder eben nicht. Ein Anästhesist bekommt beispielsweise einen Vorschlag für die Medikamentenvergabe, basierend auf Informationen über den Patienten, die Operation und vergangene Erfahrungswerte. Ein Fehler des Systems würde den Patienten gefährden, jedoch nur dann, wenn der Anästhesist dem Vorschlag folgt [22].

d. System zur Überwachung und Diagnose

Ein Fehler des Systems führt zum Ausbleiben einer Warnung und kann, wenn der Fehler nicht anderweitig erkannt wird, zu einer Gefahr für Personen und Umwelt werden. Versagt das Diagnosesystem einer Industriemaschine beim Auftreten fehlerhaften Verhaltens, so kann ein Ausfall dieser Diagnose zu einer Gefahr werden [23].

3. Sicherheitskritisch

Systeme, die bei einem Fehler direkt zu einer Gefährdung von Personen oder der Umwelt führen, werden als sicherheitskritisch bezeichnet.

a. Automation überwacht/korrigierbar

Wird eine Aktion ohne zusätzliche Bestätigung automatisiert ausgeführt, so führt der Fehler eines automatisierten Systems direkt zu einer Gefährdung von Personen und Umwelt. Wird das System zusätzlich durch einen Menschen überwacht und ist die Möglichkeit zur Korrektur gewährleistet, kann die Gefahr abgewandt werden. Der/die Überwacher/in bringt das System wieder unter Kontrolle, sodass Fehlertoleranz durch den Menschen hergestellt wird. Dabei ist zu beachten, dass besonders mit zunehmender Automatisierung der Prozesse und erweiterter Entlastung des Menschen dessen Fähigkeiten abnehmen, einen automatisierten Prozess zu überwachen [24]. Der sogenannte Stauassistent als Teilautomatisierung der Fahrzeugführung bildet ein solches 
sicherheitskritisches System, denn ein fehlerhaftes Systemverhalten birgt direkt eine Gefahr. Diese Gefahr wird jedoch mit der Überwachung durch den Fahrer adressiert, denn der Stauassistent ist so entwickelt, dass der Mensch fehlerhaftes Systemverhalten durch sein Eingreifen kontrolliert.

b. Automation nicht überwacht/ohne Korrekturmöglichkeit

Sicherheitstechnisch am kritischsten ist eine Automation ohne Korrekturmöglichkeit. Ein Fehler des Systems führt ohne Überwachung direkt zu einer Gefährdung und abhängig von der Situation zu einem Schaden von Personen und Umwelt. In diese Kategorie ist das vollautomatisierte Fahren einzuordnen, denn nach Definition muss der Insasse des Fahrzeugs dieses nicht mehr überwachen. Somit führt ein durch das System nicht adressierter Fehler oder ein unerwünschtes Verhalten direkt zu Gefahr und eventuellem Schaden von Personen und Umwelt.

Diese Kategorisierung und die Einordnung der autonomen Fahrt in die Kategorie nicht überwachte Automation zeigt, warum aktuell vorhandene Verfahren des Maschinellen Lernens aus sicherheitsunkritischen bzw. sicherheitsrelevanten Bereichen nicht einfach direkt übernommen werden können. Nicht ohne Grund sind die Berichte über sicherheitskritische Beispiele aus Abschn. 22.3.2 ohne Verweis auf einen nicht überwachten Einsatz.

Der Einsatz von Verfahren des Maschinellen Lernens in einer Automation, die nicht überwacht oder ohne Korrekturmöglichkeit ist, bedarf einer weiteren Einordnung, denn abhängig vom Zeitpunkt im Systemlebenszyklus eines Fahrzeugs leiten sich unterschiedliche Herausforderungen ab.

\subsubsection{Herausforderungen und Lösungsansätze in den verschiedenen Phasen des Systemlebenszyklus}

Der Fahrzeuglebenszyklus wird in diesem Bericht in die fünf Phasen Forschung, Entwicklung, Betrieb, Service sowie Nutzerwechsel/Stilllegung aufgeteilt, denn in jeder Phase warten auf den Einsatz des Maschinellen Lernens unterschiedliche Herausforderungen.

\subsubsection{Forschung}

Wird Maschinelles Lernen in der Forschung eingesetzt, geht es zumeist darum, die Machbarkeit von Verfahren zu zeigen. Die Bandbreite der Beispiele reicht von Online, Offline, Supervised, Unsupervised bis hin zu Reinforcement Learning. Als Datenbasis werden beispielhafte Trainingsdatensätze herangezogen. Gleiches gilt für die Bewertung der Leistungsfähigkeit und Robustheit der Verfahren. Diese wird innerhalb von kontrollierten und/ oder überwachten Bedingungen basierend auf beispielhaften Testdaten bzw. Testfahrten durchgeführt. Besonders kontrollierte Bedingungen und/oder der Einsatz ausgebildeter Testfahrer (Kategorie 3.a, s. Abschn. 22.4.1) machen Fehler tolerierbar, sodass eine Vielzahl von umgesetzten Beispielen existiert. Dementsprechend gehört zur Herausforderung für den Einsatz maschineller Lernverfahren nicht der Nachweis der Sicherheit. Die Her- 
ausforderung für den Einsatz von Maschinellem Lernen im Forschungsbereich des automatisierten Fahrens besteht im Zugang zu repräsentativen Daten für den späteren Einsatzbereich. Folglich stellt sich die Frage nach der Übertragbarkeit der Forschungsergebnisse auf die Systemlebenszyklen Entwicklung und Betrieb.

\subsubsection{Entwicklung}

Das Lernen während der Entwicklungsphase kann mit dem Offline-Lernen verglichen werden. Möglichst anwendungsrelevante Trainingsdaten werden gezielt gesammelt, um während der Entwicklung beispielsweise ein Modell zu lernen. Die Erfüllung der Anforderungen an die Sicherheit der Ergebnisse wird, wie bei allen anderen sicherheitsrelevanten Komponenten des Fahrzeugs auch, verifiziert und validiert, sodass die Freigabe des Fahrzeugs für die Produktion und den Einsatz erteilt werden kann. Danach verändern sich das gelernte Modell oder die Klassifizierung nicht weiter. Das Lernverfahren arbeitet nicht online bzw. nicht adaptiv, nutzt somit weitere Daten, die im Betrieb erfahren werden, nicht als Trainingsdaten, um das Modell oder die Klassifizierung zu aktualisieren. Es handelt sich somit während des Einsatzes um ein zeitinvariantes System, wodurch die bekannten Methoden für Verifikation und Validierung gültig bleiben. Allerdings ist zu beachten, dass die Ergebnisse der verschiedenen maschinellen Lernverfahren unterschiedlich gut interpretierbar sind. Beispielsweise sind gelernte Entscheidungsbäume mit begrenztem Umfang oder ein überschaubarer Satz an gelernten Regeln sehr gut interpretierbar [25] und ermöglichen somit den Einsatz von White-Box-Testverfahren [26]. Andere Verfahren wie z. B. die Random-Forest-Verfahren oder subsymbolische Neuronale Netzwerke hingegen sind für einen Tester schwer interpretierbar und stellen somit eine Black-Box dar. Für solch komplexe Komponenten bedeutet der Nachweis der Sicherheit eine größere Herausforderung, verglichen mit analytischen Modellen. Da sich, wie auch für die meisten analytischen Modelle, für Systeme mit hoher Eingangsdimensionalität das Brute-Force-Testen ${ }^{2}$ nicht eignet [27], werden Otte [25] folgend und erweiternd vier Gegenmaßnahmen in Betracht gezogen:

1. Herunterbrechen eines vieldimensionalen Problems auf niedrigdimensionale Teilmodelle, damit die Teilmodelle von Experten unabhängig interpretiert und validiert werden können,

2. Einsatz von Vergleichslösungen, die eine Bewertung der Sicherheit der gelernten Komponente ermöglichen,

3. Begrenzen der Eingangs-, Ausgangs- und Zustandsgrößen auf bestimmte Wertebereiche, wie z. B. die der Trainingsdaten. Diese Begrenzungen können statisch sein, aber auch von anderen Größen abhängen, und

4. Begrenzen der Dynamik von Eingangs-, Ausgangs- und Zustandsgrößen auf minimale, maximale, positive oder negative Änderung pro Zeit.

2 Der Brute-Force-Ansatz steht für das Ausprobieren (Testen) aller möglichen Kombinationen der das Testobjektverhalten beeinflussenden Parameter. 
Jede dieser Maßnahmen beschränkt das Potenzial des Maschinellen Lernens, um den Test des gelernten Systems zu ermöglichen.

\subsubsection{Betrieb}

Befindet sich das fertig entwickelte und produzierte Fahrzeug im Betrieb, fallen Daten über den realen Einsatzbereich an, und zwar über die statische Umwelt, andere Verkehrsteilnehmer und ihr Verhalten sowie den Nutzer und die Insassen des Fahrzeugs. Zusätzlich besitzt das Fahrzeug Daten über sein maschinelles Verhalten über der Zeit. Diese direkt vorhandenen neuen Informationen, die zuvor nicht zugänglich waren, motivieren den Einsatz von Online-Lernverfahren und somit adaptiven Systemen. Damit wird das Fahrzeug zu einem zeit- bzw. erfahrungsvarianten System. Dieser weitere Freiheitsgrad eines sich ändernden und zusätzlich nicht überwachten Systems ergibt eine besondere Herausforderung für das Testen und die Absicherung, die schon für zeitinvariante, autonome Systeme bisher nicht gelöst wurde (s. Kap. 21). Grundsätzlich lassen sich zwei Möglichkeiten aufzeigen, wie ein System, das sich während des Betriebs verändert, abzusichern wäre. Entweder wird die Adaptivität auf einen klar begrenzten, einhüllenden Bereich eingeschränkt, wie etwa bei Adaptive Transmission Control-Strategien [28]. Dort sind Eingangs-, Ausgangs- und Zustandsraum auf wenige Parameter begrenzt [29], sodass aus Autorensicht eine Verifikation und Validierung während der Entwicklungsphase möglich scheint. Widerspricht diese Begrenzung dem Einsatzzweck von Maschinellem Lernen während des Betriebs, ist eine Online-Überprüfung des sich ändernden, zeitvarianten und komplexen Systems notwendig [30]. Dafür wiederum bieten sich die zwei folgenden Ansätze an [30]:

\section{Runtime Verification \& Validation}

Im Gegensatz zum klassischen Vorgehen der Verifikation und Validierung während des Entwicklungsprozesses durch den Entwickler wendet das System während des Betriebs Verifikations- und Validierungs-Methoden an [27, 30, 31]. Prinzipiell wird der Adaptionsprozess als Feedback-Loop gesehen. In Abb. 22.1 sind die vier Schritte Beobachten, Ana-

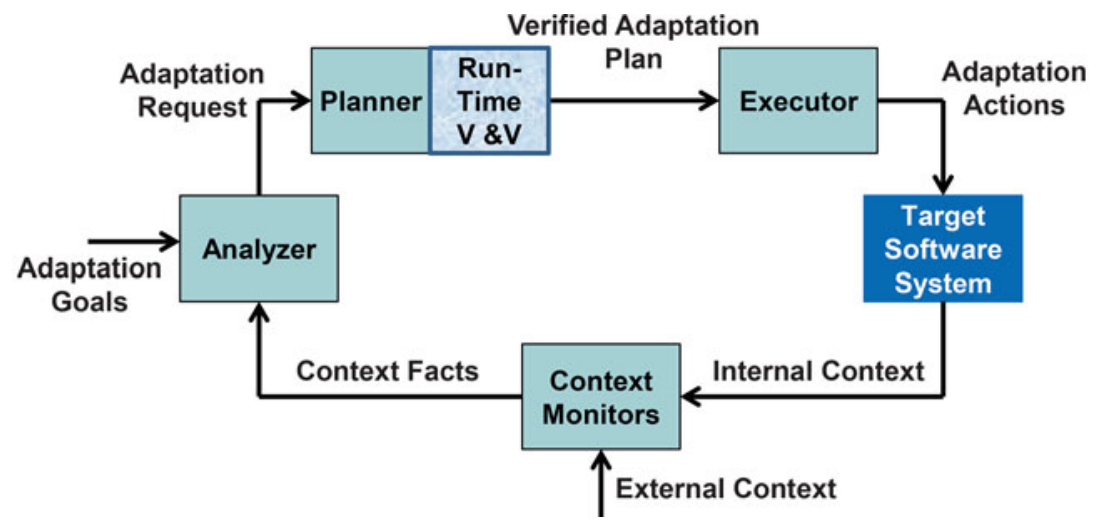

Abb. 22.1 Adaptionsprozess als Feedback-Loop mit Runtime Verification \& Validation (nach [31]) 
lysieren, Planen und Ausführen dargestellt, die nach Tamura et. al. [31] für eine strukturierte Betrachtung des Adaptionsprozesses notwendig sind. Diese Darstellung lässt sich direkt auf Online-Lernverfahren übertragen. Wird in diesem Prozess eine Adaption des Systems ermittelt, ist diese mit Verfahren der Runtime Verification \& Validation zu prüfen, bevor sie auf die ausführende Software angewandt wird. Dabei wird überprüft, ob durch die geplanten Änderungen das System den sicheren Bereich (viability zone) nicht verlässt. Konkret bedeutet dies für das autonome Fahren, dass vor Implementation der Änderung online zu überprüfen ist, ob die aktualisierte Fahrzeugführung die Anforderungen an Sicherheit erfüllt.

Die dabei eingesetzten Verfahren wie beispielsweise Modellprüfung (model checking) oder automatische Theorembeweiser (theorem proving) stoßen dabei, wie in Kap. 21 beschrieben, an ihre Grenzen. Auch der Einsatz von Software-in-the-Loop-Verfahren wäre denkbar, jedoch ist fraglich, ob ausreichend Rechenleistung für solche Verfahren in Serienfahrzeugen vorhanden ist.

2. Validierung und Verifikation durch Monitoring und Fehlertoleranz

Ist eine Überprüfung der Sicherheit des Systems vor Aktualisierung der Software nicht möglich, kann ein Fehler (fault) auftreten und zu einem Ausfall (failure) führen und somit zu einer Gefahr werden. Um dies zu verhindern, ist das System fehlertolerant auszulegen. Ein solches fehlertolerantes System bedarf grundsätzlich zweier Komponenten [32]: zum einen einer Überwachung (monitoring) der Zustände und des Verhaltens des Systems, um basierend auf einer Bewertung zu entscheiden, ob ein Fehler (fault) vorhanden ist. Zum anderen wird eine Redundanz benötigt, an die im Fehlerfall „übergeben“ werden kann. Abb. 22.2 zeigt schematisch diesen Aufbau. Dieses Prinzip entspricht dem menschlichen Überwacher, der im Fehlerfall eines teilautomatisierten Systems die Fahrzeugführung übernimmt.

Die durchgeführte Betrachtung zeigt, dass Maschinelles Lernen während des Betriebs besonders für die Absicherung des sicheren Verhaltens eine Herausforderung darstellt. Beide Verfahren, sowohl die Runtime Verification \& Validation als auch die Verifikation und Validierung durch Monitoring benötigen ein Maß für sicheres Fahren. Ansätze für ein solches Maß werden in Abschn. 22.4.3 vorgestellt.

Einen weiteren Ansatz liefert an dieser Stelle der Vergleich mit dem menschlichen Lernen. Die Teilnehmer des Straßenverkehrs akzeptieren, dass ein Mensch ohne weitere

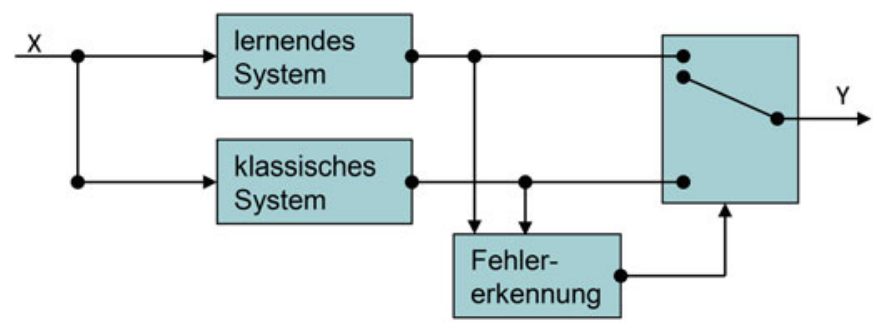

Abb. 22.2 Dynamische Redundanz (nach [32]) 
Kontrolle basierend auf seinen Maßen für die Fahrzeugführung lernt und sein Verhalten anpasst. Die Überprüfung, ob das angepasste Verhalten den Regeln des Straßenverkehrs genügt, erfolgt nicht direkt, sondern sporadisch über Verkehrs-, oder Geschwindigkeitskontrollen der Polizei. Zusätzlich melden andere Verkehrsteilnehmer schwerwiegendes Fehlverhalten wie z. B. Unfälle, sodass der Mensch dies für den Lernprozess zurückgemeldet bekommt. Übertragen auf das technische System würde dies bedeuten, dass Maschinelles Lernen direkt zu einer Anpassung des Verhaltens führt. Der Test bzw. die Kontrolle, ob diese Anpassung legitim war, erfolgt nachträglich durch andere Verkehrsteilnehmer, die Polizei oder eine spezielle Kontrollinstanz. Dieser Ansatz reduziert die Anforderungen an die Online-Verifikation und -Validierung, denn die Fähigkeiten der anderen Verkehrsteilnehmer werden mit einbezogen. Geschieht der Test erst nach Aktualisierung und Änderung der Funktionen und besteht zusätzlich keine direkte Eingriffsmöglichkeit (Steuerung), wird durch den Betrieb der aktualisierten, aber nicht getesteten Funktion ein erhöhtes Risiko eingegangen.

\subsubsection{Service}

Neben dem Maschinellen Lernen während der Entwicklung und des Betriebs gibt es im Systemlebenszyklus eine weitere Phase, in der gelernt werden kann. Im Rahmen von Serviceangeboten können vom Fahrzeug aufgezeichnete Trainingsdaten heruntergeladen und Funktionen des Fahrzeugs aktualisiert werden. Dafür wird nicht zwangsweise die physikalische Anwesenheit des Fahrzeugs benötigt [33]. Durch dieses Vorgehen wird der Feedback-Loop aus Abb. 22.1 geöffnet. Trainingsdaten und geplante Adaptionen können offline entsprechend einem weiteren Entwicklungsschritt getestet werden, sodass nach dem Nachweis der Sicherheit das Softwaresystem zeitversetzt aktualisiert wird. Da für diese Methoden des Maschinellen Lernens auch personenbezogene Daten das Fahrzeug verlassen könnten, ist zusätzlich zur Sicherheit im Sinne des englischen Begriffs Safety auch der Security-Aspekt zu beachten. Für eine weitergehende Betrachtung wird auf Kap. 24 verwiesen.

\subsubsection{Nutzerwechsel / Fahrzeugstilllegung}

Besitzt das Fahrzeug die angestrebte Möglichkeit, die Ausführung der Fahrzeugführung für einen Nutzer zu personalisieren oder für ein Einsatzgebiet zu optimieren, dann sollten diese erlernten Fähigkeiten bzw. das erlernte Wissen bei einem Nutzerwechsel bzw. einer Fahrzeugstillegung dem Nutzer und nicht dem Fahrzeug zugewiesen sein. Diese Fähigkeit wird dann besonders interessant, wenn sich beispielsweise beim Vehicle-on-Demand (s. Kap. 2) die Besitzverhältnisse verändern und der Nutzer nicht ein bestimmtes Fahrzeug, sondern nur dessen Mobilitätsdienstleistung erwirbt. Prinzipiell stellt die Übertragung des Wissens für ein technisches System keine Schwierigkeit dar, es ist eher die Stärke des künstlichen Systems, Wissen ohne den langwierigen Lernprozess übertragen zu können. Diese Betrachtung wird in Abschn. 22.5 weiter ausgeführt. 


\subsubsection{Maße sicheren Fahrens}

Wie in den vorherigen Abschnitten beschrieben, wird sowohl für das generelle Verfahren des Maschinellen Lernens als auch für die Verifizierung und Validierung während des Betriebs die Bewertung der Fahrzeugführung in Bezug auf die Sicherheit benötigt. Zunächst kann natürlich nachträglich bewertet werden, ob ein Unfall stattgefunden und mit welcher Aufprallgeschwindigkeit, Aufprallenergie und Unfallkonstellation sich dieser ereignet hat. Dieses Maß hat den Nachteil, dass der Unfall nach Möglichkeit gar nicht auftreten sollte. Daraus folgt, dass für ein sicheres System der Unfall ein extrem seltenes Ereignis sein wird und somit kaum zum Lernen geeignet ist.

Gesucht ist eine Bewertung, die bereits vor Übertreten der fahrphysikalischen Grenzen eine unsichere Fahrt klassifiziert. Dafür wird nach [34, 35] in der Gefahrenbewertung zwischen deterministischem und stochastischem Verfahren unterschieden.

\subsubsection{Deterministische Verfahren zur Gefahrenbewertung}

Nach [36] wird zusätzlich zwischen Identifikatoren aus der Fahrdynamik und Identifikatoren aus dem Abstand unterschieden. Die am einfachsten zu ermittelnden Werte mit jedoch begrenzter Aussagekraft sind Grenzwerte für Längs- und Querbeschleunigungen sowie die Gierrate. Bei der 100 Cars Study [37] wurden u. a. Längsbeschleunigungen größer als 0,7 $g$ als Trigger für die Detektion von unsicheren Situationen herangezogen. Die Identifikation einer kritischen Situation ausschließlich bezogen auf Ego-Fahrzeug-Größen ist nicht hinreichend, denn andere Verkehrsteilnehmer können auch ein stehendes Fahrzeug in gefährliche Situationen überführen. Es ist leicht nachzuvollziehen, dass die Sicherheit des Fahrzeugs auch durch andere Verkehrsteilnehmer im Umfeld des Fahrzeugs beeinflusst wird.

Wird das Umfeld für den Anfang zunächst auf den Längsverkehr auf einem Fahrstreifen reduziert, ermöglichen die Time-To-Collision TTC bzw. deren Kehrwert eine Aussage über die Sicherheit der Situation. Beispielsweise definiert die ISO 22839 (Forward vehicle collision mitigation systems) als Zeit

$$
T T C=\frac{x_{c}}{v_{r}}
$$

die vergeht, bis das Ego-Fahrzeug mit dem Objekt im Abstand $x_{c}$ kollidiert, angenommen die Relativgeschwindigkeit $v_{r}=\left(v_{\text {ego }}-v_{o b j}\right)$ bliebe konstant. Diese Größe kann sowohl für das vorausfahrende als auch das nachfolgende Fahrzeug angewandt werden. Aus der TTC wird bei Chan [38] ein Kritikalitätsmaß definiert, für das angenommen wird, dass die Schwere eines Unfalls mit dem Quadrat der Geschwindigkeit einhergeht:

$$
\text { Criticality Index }=\frac{v^{2}}{T T C} \text {. }
$$


Bleibt die Geschwindigkeit zwischen den Fahrzeugen nicht konstant, wird die Enhanced TTC angewandt. In der ISO 22839 wird diese hergeleitet zu

$$
E T T C=\frac{v_{r}-\sqrt{v_{r}^{2}-2 \cdot a_{r} \cdot x_{c}}}{a_{r}} .
$$

Diese Gleichung gilt nur, solang die relative Beschleunigung $a_{r}=\left(a_{o b j}-a_{\text {ego }}\right)$ konstant bleibt. Verzögert ein Fahrzeug in den Stillstand, ist die Gleichung anzupassen (s. dazu [39]). Bei diesen Werten handelt es sich um Bewertungen eines Zustandes, entsprechend einer Situationsaufnahme. Um die situationsübergreifende Bewertung der Fahrsicherheit zu ermöglichen, werden von [40] folgende Verfahren empfohlen:

- Number Of Conflicts (NOC),

- Time Exposed TTC (TET),

- Time Integrated TTC (TIT).

Die Namen der Verfahren sprechen für sich, sodass an dieser Stelle nicht weiter darauf eingegangen, sondern auf $[40,41]$ verwiesen wird. Weitere einfache Maße sind die Zeitlücke (time headway)

$$
t_{h}=\frac{x_{c}}{v_{\text {ego }}}
$$

sowie die notwendige Verzögerung $a_{\text {req }}$, um einen Auffahrunfall zu vermeiden. In [41-43] sind weitere Ansätze basierend auf den zuvor erläuterten zu finden.

Werden nun zusätzlich Fahrzeuge, Objekte und Beschleunigungen in lateraler Richtung betrachtet, beispielsweise für die Untersuchung von Kreuzungssituationen, müssen die Maße für eine Sicherheitsbewertung erweitert werden. Der wohl einfachste Ansatz ist die Post-Encroachment Time (PET). Diese zeitliche Größe ist von [44] definiert als "time between the moment that the first road user leaves the path of the second and the moment that the second road user reaches the path of the first".

Aufgrund der Betrachtung der lateralen Richtung stehen nicht mehr nur zwei ObjektFahrzeuge in Beziehung zu dem Ego-Fahrzeug, sondern weitere Objekte. Diese können statisch, aber auch dynamisch aus prinzipiell allen Richtungen der Fahrzeugebene Einfluss auf die Sicherheitsbewertung haben. Tamke [45] liefert hierfür einen Ansatz, indem gegenüber allen Objekten in der Umgebung des Ego-Fahrzeugs beispielsweise mit der euklidischen Norm der Abstand sowie die zeitliche Ableitung des Abstands bestimmt wird. Basierend auf diesen Größen wird die TTC bestimmt, indem mithilfe einer Verhaltensprädiktion die Zeit bis zum Kontakt der Fahrzeughüllen ermittelt wird. Dieser Ansatz wird an dieser Stelle noch den deterministischen Verfahren zugeordnet, da das Verhalten des Ego-Fahrzeugs sowie der dynamischen Objekte dem ,,constant turn and constant acceleration-Ansatz" folgen. Der Ansatz an sich liefert jedoch die Möglichkeit, die Prädiktion des Verhaltens auch nichtdeterministisch zu gestalten. 
Deterministische Verfahren sind für eine nachträgliche Bewertung der Sicherheit eines erlebten Manövers oder einer Situation geeignet. Wird jedoch auf eine Online-Bewertung von aktuellen Situationen und die Prädiktion auf einen Zeithorizont größer $1 \mathrm{~s}$ gezielt, dann ist die Anwendung per se fehlerhaft, solang in der Situation Unsicherheiten enthalten sind. Mit Unsicherheiten ist gemeint, dass die Entwicklung der Situation über der Zeit nicht nur einen möglichen deterministischen Verlauf hat, wie oft bei „constant turn and constant acceleration“-Modellen der Einfachheit angenommen wird, sondern viele verschiedene Situationen eintreten können. Beispielsweise könnte das bei der Berechnung der TTC vorausfahrende Fahrzeug auch beschleunigen anstatt weiter zu verzögern und somit eine kritische Situation entschärfen. Um diese Unsicherheiten mit in die Gefahrenbewertung einfließen zu lassen, werden im Folgenden stochastische Verfahren kurz vorgestellt.

\subsubsection{Stochastische Verfahren zur Gefahrenbewertung}

Die Gefahrenbewertung einer einfachen Vorbeifahrt zweier Fahrzeuge, wie in Abb. 22.3 dargestellt, motiviert anschaulich den Einsatz stochastischer Verfahren. Würde von einem deterministischen Verhalten und der Extrapolation von Geschwindigkeit und Lenkwinkel ausgegangen werden, würde eine Vorbeifahrt keine Gefahr bedeuten, denn die Trajektorien bzw. die Aufenthaltsbereiche der Fahrzeuge kreuzen sich nicht. Dies sieht anders aus, wenn Unsicherheiten in Betracht gezogen werden. Wenn das menschgeführte Fahrzeug (s. Abb. 22.3, unterer Fahrstreifen, schwarz) nicht dem Fahrstreifen folgt, können für das autonome Fahrzeug (s. Abb. 22.3, oberer Fahrstreifen, orange) und für das Umfeld Gefahren entstehen.
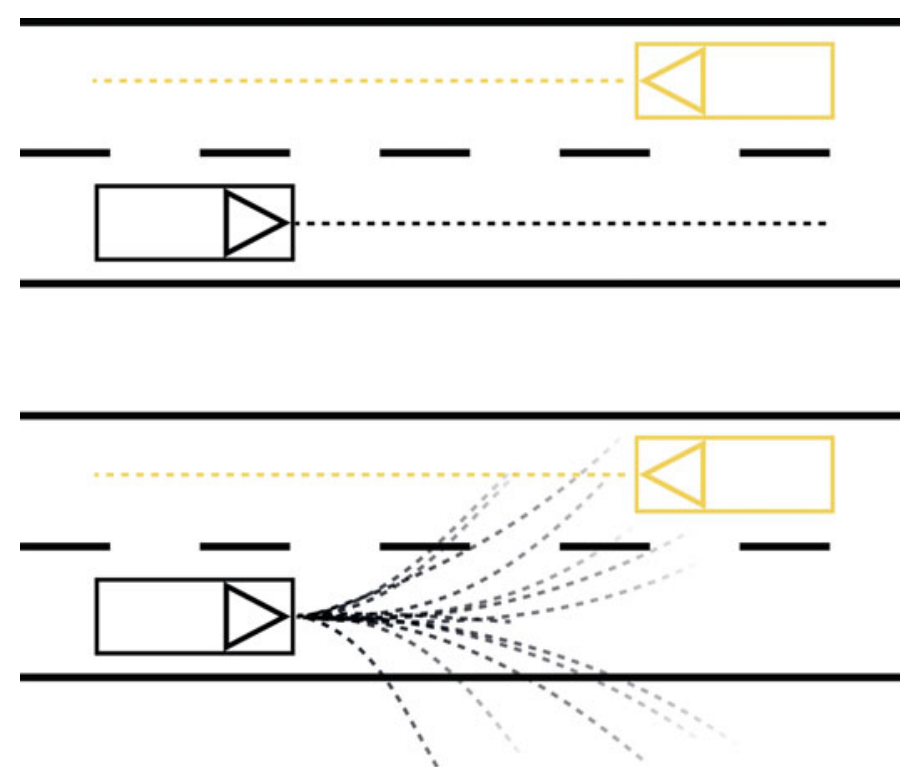

Abb. 22.3 Vergleich Situationsprädiktion einer Vorbeifahrt: deterministisch (oben), stochastisch (unten) 
Das damit einhergehende Risiko folgt aus der Wahrscheinlichkeit für einen Unfall sowie aus der potenziellen Schwere dieses Unfalls. Bei beiden Werten handelt es sich um Unbekannte, die es möglichst genau zu schätzen gilt.

Dabei ist der prinzipielle Ansatz [46] für die Ermittlung der Wahrscheinlichkeit, die Trajektorien von Ego-Fahrzeug und Objekten im Umfeld, basierend auf gemessenen $\mathrm{Zu}-$ standsgrößen, zu prädizieren. Aufgrund der erwähnten Unsicherheiten durch den menschlichen Fahrer, durch Sensoren und Aktoren und einer Interaktion zwischen den Objekten gibt es nicht nur die eine Trajektorie, sondern eine Wahrscheinlichkeitsverteilung der Zustände aller Objekte über der Zeit. Überdecken sich die möglichen Aufenthaltsbereiche der Objekte, besteht eine Wahrscheinlichkeit für einen Unfall. Die möglichen Zustände der Objekte folgen aus eingesetzten dynamischen Modellen und aus definierten Grenzwerten für dynamische Größen. Bei [34] wird dafür ein Einspurmodell für Fahrzeuge eingesetzt, um einen Kompromiss zwischen Prädiktionsgenauigkeit und Rechenaufwand zu finden. Zusätzlich werden die dynamischen Größen wie Beschleunigung und Lenkrate auf - nach Einschätzung des Autors [34] - unkritische und typische Werte begrenzt. In [34, 47] findet sich eine Übersicht über alternative Methoden. Allen Methoden ist gemein, dass die Dynamiksimulation eines Fahrzeugs nicht analytisch erfolgen kann [45], sodass numerische Methoden mit einhergehender Diskretisierung und Vereinfachung einzusetzen sind.

Das Einbeziehen der Schwere in die Gefahrenbewertung wird bei [48] durch eine relative Bewertung jedes Unfalls anhand des unelastischen Stoßes angenähert. Dies entspricht dem auf der Potential Collision Energy (PCE) basierenden Ansatz [40]. Darüber hinaus wurden jedoch keine Ansätze gefunden, die sowohl Schwere als auch Wahrscheinlichkeit in Kombination bestimmen. Ein Grund dafür sind auf der einen Seite ungenaue analytische Regressionsmethoden [49] und auf der anderen Seite rechenintensive Finite-ElementeMethoden (FEM), die aktuell auch in anderen Bereichen für die Unfallschwerebestimmung eingesetzt werden [50]. Meier et al. [49] liefern hierfür einen neuen Ansatz, der auf symbolischer Regression basiert. Mithilfe einer Datenbank von Crashsituationen (gewonnen durch FEM-Berechnungen) werden Regressionsfunktionen gelernt, die für eine Situation die zugehörige Unfallschwere innerhalb von wenigen Millisekunden vorhersagen. Dafür werden Precrash-Informationen wie Fahrzeugmasse, Geschwindigkeiten, Kollisionspunkt und Kollisionswinkel genutzt. Nachteil dieses Ansatzes ist die bedingte Interpretierbarkeit, da das Regressionsmodell nicht aus physikalischen Größen gebildet wird. Wenn dieser Ansatz eine valide Prädiktion der Schwere liefert, könnte damit die Risikobewertung um eine Schwerebewertung ergänzt werden.

Für die Bewertung der Sicherheit der adaptiven automatisierten Fahrzeugführung sind die vorgestellten Verfahren aktuell aus folgenden Gründen noch nicht umfassend geeignet. Zum einen basieren sämtliche Ansätze auf einer Reihe von Vereinfachungen wie beispielsweise der Vernachlässigung von Wetterbedingungen, der Vereinfachung der Fahrdynamik sowie der fehlenden Betrachtung von Sensorunsicherheiten. Zum anderen bieten die aktuellen Verfahren, wie vorgestellt wurde, noch keine validierte und kombinierte Ermittlung der Wahrscheinlichkeiten und Schweren von Unfällen. Eine allgemeine Definition und Bewertung der Sicherheit der Fahrzeugführung ist somit aktuell noch nicht gegeben. 
Allerdings liefert die voranschreitende Fahrzeugautomatisierung begünstigende Faktoren. Diese sind zum einen das Entfernen der Unsicherheit des Fahrers (Ego-Fahrzeug). Zwar ist menschliches Verhalten durch die anderen Verkehrsteilnehmer weiterhin vorhanden, die Trajektorie des Ego-Fahrzeugs ist jedoch innerhalb der Regelgüte bekannt. Außerdem steigt die Sensorleistungsfähigkeit aktueller Fahrzeuge, sodass Unsicherheiten über die Objektzustände abnehmen. Ferner werden zusätzliche Informationen über das Umfeld per V2X-Kommunikation ausgetauscht und damit die Informationsgrundlage für eine Gefahrenbewertung in Qualität und Quantität verbessert.

\subsection{Die Automation als Teil eines lernenden Kollektivs}

Die Betrachtung des lernenden Fahrzeugs beschränkte sich in diesem Kapitel bisher auf den Systemlebenszyklus eines speziellen Fahrzeugs. Was die Fahrzeugautomatisierung jedoch implizit mit sich bringt, ist die Vervielfältigung der Hard- und Software durch die angestrebte Serienproduktion auf ein ganzes Kollektiv von Fahrzeugen. Entsprechend würden sich Fahrzeuge im Straßenverkehr bewegen, die gleiche Fähigkeiten aufweisen. Dies hat auf der einen Seite den Nachteil, dass Fehlverhalten, Ausfälle und Unfallarten nicht nur ein Fahrzeug, sondern gleich das gesamte Kollektiv betreffen können. Auf der anderen Seite eröffnet es einen weiteren Freiheitsgrad für adaptive Systeme. Eine Datenaustauschmöglichkeit vorausgesetzt, ergibt sich die Möglichkeit zum Kollektiven Lernen während des Betriebs. Dabei lassen sich prinzipiell zwei Ansätze unterscheiden [51]: das agentenbasierte Maschinelle Lernen und das Maschinelle Lernen mit Agenten. Bei dem agentenbasierten Lernen (auch oft agentenbasierte Schwarmintelligenz genannt) baut sich das lernende kognitive System aus einer Vielzahl von vernetzten Agenten mit begrenzter kognitiver Leistungsfähigkeit auf, dabei wird oft das Verhalten von Tieren wie Ameisen oder Bienen als Beispiel herangezogen [52]. Im Gegensatz dazu besteht das Maschinelle Lernen mit Agenten ebenfalls aus mehreren Agenten, die jedoch die in Abschn. 22.3 beschriebenen Verfahren anwenden. Diese zwei Ansätze unterscheiden sich in den grundlegenden Schritten des Maschinellen Lernens hinsichtlich

- kollektiver Erfahrungsgenerierung,

- kollektiver Leistungsbewertung und

- Ableitung von gelernten Modellen und von Wissen.

Grundsätzlich können, sobald Daten von der realen Situation erzeugt wurden, die Ansätze angewandt werden. Dabei stellt sich die Frage, an welcher Stelle eines Kollektivs aus den Daten relevante Informationen erzeugt werden, diese im Sinne des Lernproblems und des Leistungsmaßes bewertet werden und basierend darauf Lernverfahren eingesetzt werden. Nicht zuletzt aus Gründen der begrenzten Bandbreite für die Datenübertragung wird es notwendig sein, bereits verarbeitete Daten oder sogar die gelernten Modelle und das Wissen zu übertragen, anstelle von Sensorrohdaten. Solang die beteiligten Agenten/Fahrzeuge 
dabei einer Serie und einem Softwarestand angehören, folgen Anforderungen an die Integrität der übertragenen Informationen, jedoch nicht an die Kompatibilität und Vertrauenswürdigkeit zwischen den Agenten. Sogenannte homogene Teams existieren, bei denen untereinander bekannt ist, wie und was gelernt wird. Ist das Ziel jedoch, die Datenbasis zu erweitern, auf der Lernverfahren angewandt werden, könnten auch Fahrzeuge anderen Softwarestands oder sogar anderer Hersteller untereinander vernetzt werden. Es entsteht ein Kollektiv aus heterogenen Fahrzeugen, bei denen eventuell unterschiedliche Verfahren des Maschinellen Lernens eingesetzt werden und die Wissensrepräsentation ebenfalls heterogen ist. Gifford [53] zeigt für Beispiele aus anderen Bereichen, wie prinzipiell mit solchen Kollektiven umgegangen werden könnte. Grundsätzlich existieren neben den Fahrzeugrobotern noch weitere Agenten, wie beispielsweise Smartphones oder zukünftig auch Serviceroboter, die ebenfalls Daten erzeugen und damit vollkommen andere Informationsbereiche abdecken, als sie Fahrrobotern zugänglich sind. Die aktuell vorstellbare letzte Stufe der Konnektivität und mit Abstand die größte Datenbasis liefert das Internet. Das autonome Fahrzeug als web-enabled device ermöglicht eine Vielzahl von Anwendungen und Funktionen im positiven wie auch im negativen Sinne. Nicht zuletzt das Watson-Projekt von IBM zeigt, dass Teile des im Internet abgelegten Wissens auch für Maschinen verständlich sind. Informationen, die von einer beliebigen Instanz über das Internet zugänglich gemacht werden, müssten somit nicht erst erfahren werden, um das Verhalten des autonomen Fahrzeugs zu beeinflussen. Durch den Zugriff auf beliebige (nicht autorisierte bzw. anonyme) Quellen droht jedoch eine Herausforderung für die Sicherheit sowohl im Sinne der Verkehrssicherheit (safety) als auch der Datensicherheit (security, s. Kap. 24).

\subsection{Fazit}

Das Maschinelle Lernen findet in der Forschung aktuell großes Interesse, da die Qualität und Quantität vorhandener Daten stetig zunimmt und zusätzlich die Fahrzeugautomatisierung Fragen aufwirft, die nur bedingt mit herkömmlichen analytischen Ansätzen gelöst werden können. Bei der Übertragung der Ergebnisse von der Forschung auf die Entwicklung der Serienfunktionen für die autonome Fahrzeugführung stellt jedoch vor allem der Nachweis der Sicherheit für den Einsatz im nicht überwachten sicherheitskritischen System ohne Korrekturmöglichkeit eine Herausforderung dar. Deshalb finden sich bereits in aktuellen Serienfahrzeugen nach Wissensstand der Autoren nur gelernte Modelle, die sich nach dem Test und der Freigabe nicht mehr ändern. Systeme wie die Adaptive Transmission Control sind bei dieser Betrachtung aufgrund der geringen adaptiven Parameter in klar begrenzten Wertebereichen ausgeschlossen. Allerdings öffnet gerade das Lernen im Betrieb und somit die Adaption einen weiteren Freiheitsgrad für die Automation. Diesen Freiheitsgrad zu nutzen, motiviert neben der Kompensation des Wegfalls der Anpassungs- und Lernfähigkeit des Menschen sowie der Individualisierung der Fahrzeugführung zusätzlich die Möglichkeit, die autonome Fahrt zu optimieren. Das Kapitel zeigt auf, dass bei der 
Anwendung des Maschinellen Lernens für die Fahrzeugautomatisierung während des Betriebs sowohl die Verkehrssicherheit (safety) als auch die Datensicherheit (security) mit hoher Priorität zu betrachten sind. Für die Bewertung der Verkehrssicherheit respektive des Risikos fehlt aktuell noch ein valides Maß. Deshalb liegt nahe, dass der Einsatz von adaptiven Maschinellen Lernverfahren während des Betriebs zunächst eine fehlertolerante Auslegung mit redundanten herkömmlichen Systemen erfordert, bei denen die herkömmlichen Systeme zur Bewertung der Verkehrssicherheit dienen. Somit ist zu erwarten, dass Maschinelles Lernen während des Betriebs (adaptive Systeme) die Fahrzeugautomatisierung zunächst nur innerhalb eines vorgegebenen Rahmens der herkömmlichen Systeme optimieren wird.

Aufgrund der aufgezeigten Herausforderungen hinsichtlich des Nachweises eines sicheren Verhaltens des zeitvarianten adaptiven Systems erscheint es notwendig, intensiv weiter an einer Runtime-Verifikation und -Validierung zu forschen. Gleiches gilt für die zuvor angesprochenen eingesetzten nicht-adaptiven Systeme und deren Nachweis der Sicherheit. Zwar existiert eine Vielzahl an Literatur über bereits erfolgreich eingeführte Beispiele, jedoch kommen diese zumeist aus anderen Bereichen, die keine vergleichbaren Anforderungen an ein Produkt stellen.

In diesem Beitrag wurde die Frage der Datensicherheit (security) nur am Rande diskutiert und stattdessen auf Kap. 24 verwiesen. Ansätze des Lernens benötigen Daten und somit Informationen über Insassen, Fahrzeug und Umwelt. Somit kommt dem Datenschutz eine der Verkehrssicherheit (safety) gleiche Relevanz zu. Anzumerken ist, dass die Umwelt natürlich auch die Personen in dieser und somit deren Datenschutz beinhaltet. Die Qualität und Quantität der für die Fahrzeugautomation benötigten Sensoren ist auf der einen Seite Antreiber des Maschinellen Lernens, aber auf der anderen Seite jedem Datenschützer suspekt. Eine besondere Eigenschaft der Fahrzeugsensoren ist zusätzlich, dass diese aktuell zu einem Großteil nicht physikalisch abgedeckt werden, wenn das Fahrzeug oder die Funktion nicht aktiv ist. Mit dem Einsatz des Maschinellen Lernens sollte dementsprechend direkt auch die Datensicherheit angesprochen werden.

Nichtdestotrotz bringt gerade der Einsatz von vernetzten Agenten und lernenden Systemen Vorteile, deren Auswirkungen nicht abzuschätzen sind. Kollektiv lernende Agenten müssen sich vorhandenes Wissen nicht langwierig aneignen, sondern können es per Datenübertragung (copy \& paste) an nächste Fahrzeug- bzw.- Softwaregenerationen übergeben. Dies, verbunden mit einem Zugriff auf die große Menge von elektronisch erfassten Informationen, besitzt das Potenzial, die Fahrzeugführung, den Straßenverkehr und somit das ganze (Mobilitäts-)Verhalten der Menschen zu verändern. Dabei sind die Erkenntnisse, die für die Fahrzeugführung gewonnen werden, in gleichem Maße für die Forschung an medizinischen Robotern sowie Haushaltsrobotern mit Menschenkontakt von Interesse. Aufgrund der ähnlichen Rahmenbedingungen gilt Gleiches auch umgekehrt, was für eine enge Zusammenarbeit zwischen Fahrzeugtechnik und Robotik spricht. 


\section{Literatur}

1. Rasmussen, J.: Skills, Rules, and Knowledge; Signals, Signs, and Symbols, and Other Distinctions in Human Performance Models. IEEE Transactions On Systems, Man, and Cybernetics SMC-13(3), 257-266 (1983)

2. Donges, E.: Fahrerverhaltensmodelle. In: Winner, Hakuli, Wolf (Hrsg.) Handbuch Fahrerassistenzsysteme, pp. 15-23 (2011)

3. Oswald, W.D.: Automobilismus und die ,gefährlichen Alten“. In: G. Schmidt (Hrsg.) Technik und Gesellschaft. Automobil und Automobilismus, vol. 10, pp. 183-195 (1999)

4. Williams, A.F.: Teenage drivers: patterns of risk. Journal of safety research 34(1), 5-15 (2003)

5. Burgard, E.: Fahrkompetenz im Alter: Die Aussagekraft diagnostischer Instrumente bei Senioren und neurologischen Patienten. Dissertation, LMU (2005)

6. Funk, W., Grüninger, M., Dittrich, L., Goßler, J., Hornung, C., Kreßner, I., Libal, I., Limberger, S., Riedel, C., Schaller, S.: Begleitetes Fahren ab 17 - Prozessevaluation des bundesweiten Modellversuchs. Berichte der Bundesanstalt für Straßenwesen. Unterreihe Mensch und Sicherheit.(213) (2010)

7. Mitchell, T.M.: Machine Learning. McGraw-Hill series in computer science. McGraw-Hill, New York (1997)

8. Breiman, L.: Statistical Modeling: The Two Cultures (with comments and a rejoinder by the author). Statist. Sci., 199-231 (2001)

9. Carbonell, J., Michalski, R., Mitchell, T.: An Overview of Machine Learning. In: Michalski, R., Carbonell, J., Mitchell, T. (Hrsg.) Machine Learning. Symbolic Computation, pp. 3-23. Springer Berlin Heidelberg (1983)

10. Ertel, W.: Grundkurs Künstliche Intelligenz. Eine praxisorientierte Einführung, 3rd edn. Lehrbuch. Springer Fachmedien, Wiesbaden (2013)

11. Sewell, M.: Machine Learning. http://machine-learning.martinsewell.com/ (zuletzt geprüft 15.07.2014) (2009)

12. Sammut, C. (ed.): Encyclopedia of machine learning. 78 tables. springer reference. Springer, New York (2011)

13. Shaoning Pang, Nikola Kasabov: Inductive vs transductive inference, global vs local models: SVM, TSVM, and SVMT for gene expression classification problems - Neural Networks. Proceedings. 2004 IEEE International Joint Conference on (2004)

14. Russell, S., Norvig, P., Intelligence, A.: A modern approach. Artificial Intelligence. Prentice-Hall, Egnlewood Cliffs 25 (1995)

15. March, J.G.: Exploration and exploitation in organizational learning. Organization science 2(1), 71-87 (1991)

16. Nusser, S.: Robust Learning in Safety-Related Domains. Machine Learning Methods for Solving Safety-Related Application Problems, Otto-von-Guericke-Universität Magdeburg (2009)

17. Nusser, S., Otte, C., Hauptmann, W., Leirich, O., Krätschmer, M., Kruse, R.: Maschinelles Lernen von validierbaren Klassifikatoren zur autonomen Steuerung sicherheitsrelevanter Systeme. at-Automatisierungstechnik Methoden und Anwendungen der Steuerungs-, Regelungsund Informationstechnik 57(3), 138-145 (2009)

18. Pomerleau, D.: Neural Network Vision for Robot Driving. In: Hebert, M., Thorpe, C., Stentz, A. (Hrsg.) Intelligent Unmanned Ground Vehicles, vol. 388. The Springer International Series in Engineering and Computer Science, pp. 53-72. Springer US (1997)

19. Gusikhin, O., Rychtyckyj, N., Filev, D.: Intelligent systems in the automotive industry: applications and trends. Knowl Inf Syst 12(2), 147-168 (2007). doi: 10.1007/s10115-006-0063-1

20. DIN 31000:2011-05: Allgemeine Leitsätze für das sicherheitsgerechte Gestalten von Produkten 
21. Deng, L., Li, X.: Machine Learning Paradigms for Speech Recognition: An Overview. IEEE Trans. Audio Speech Lang. Process. 21(5), 1060-1089 (2013). doi: 10.1109/TASL.2013.2244083

22. Caelen, O., Bontempi, G., Barvais, L.: Machine learning techniques for decision support in anesthesia. In: Artificial Intelligence in Medicine, pp. 165-169. Springer (2007)

23. Widodo, A., Yang, B.-S.: Support vector machine in machine condition monitoring and fault diagnosis. Mechanical Systems and Signal Processing 21(6), 2560-2574 (2007). doi: 10.1016/j. ymssp.2006.12.007

24. Bainbridge, L.: Ironies of automation. Automatica 19(6), 775-779 (1983). doi: 10.1016/00051098(83)90046-8

25. Otte, C.: SCI 445 - Safe and Interpretable Machine Learning: A Methodological Review. In: Moewes, C., Nürnberger, A. (Hrsg.) Computational intelligence in intelligent data analysis. Studies in computational intelligence, vol. 445. Springer, Berlin, New York (2013)

26. Burgdorf, F.: Eine kunden- und lebenszyklusorientierte Produktfamilienabsicherung für die Automobilindustrie, KIT Scientific Publishing; Karlsruher Institut für Technologie (2010)

27. Taylor, B.J.: Methods and procedures for the verification and validation of artificial neural networks. Springer (2006)

28. Nelles, O.: Lernfähige Fuzzy-basierte Fahrstrategie für automatische Getriebe. In: Isermann, R. (Hrsg.) Modellgestützte Steuerung, Regelung und Diagnose von Verbrennungsmotoren, pp. 233-250. Springer Berlin Heidelberg (2003)

29. Cao, C.T., Kronenberg, K., Poljansek, M.: Adaptive transmission control. Google Patents. http://www.google.com/patents/US5954777 (1999)

30. Dahm, W.: Perspectives on Verification and Validation in Complex Adaptive Systems, Notre Dame University. Workshop on Verification and Validation in Computational Science (2011). Accessed 22 July 2014

31. Tamura, G., Villegas, N., Müller, H., Sousa, J., Becker, B., Karsai, G., Mankovskii, S., Pezzè, M., Schäfer, W., Tahvildari, L., Wong, K.: Towards Practical Runtime Verification and Validation of Self-Adaptive Software Systems. In: Lemos, R. de, Giese, H., Müller, H., Shaw, M. (Hrsg.) Software Engineering for Self-Adaptive Systems II, vol. 7475. Lecture Notes in Computer Science, pp. 108-132. Springer Berlin Heidelberg (2013)

32. Isermann, R.: Fault-diagnosis systems. An introduction from fault detection to fault tolerance. Springer, Berlin, New York (2006)

33. Stokar, R. von: Software-Updates Effiziente Nutzung von Connected Cars. ATZ Elektron 9(1), 46-51 (2014). doi: 10.1365/s35658-014-0387-7

34. Eugen Käfer: Situationsklassifikation und Bewegungsprognose in Verkehrssituationen mit mehreren Fahrzeugen, Universität Bielefeld (2013). Accessed 7 July 2014

35. Eidehall, A., Petersson, L.: Statistical Threat Assessment for General Road Scenes Using Monte Carlo Sampling. Intelligent Transportation Systems, IEEE Transactions on 9(1), 137-147 (2008). doi: 10.1109/TITS.2007.909241

36. Benmimoun, M., Fahrenkrog, F., Zlocki, A., Eckstein, L.: Erkennung und Klassifizierung Kritischer Fahrsituationen Mittels Fahrzeugdaten. ATZ Automobiltech Z 114(10), 820-826 (2012). doi: 10.1007/s35148-012-0485-x

37. Guo, F., Klauer, S., Hankey, J., Dingus, T.: Near Crashes as Crash Surrogate for Naturalistic Driving Studies. Transportation Research Record: Journal of the Transportation Research Board 2147(-1), 66-74 (2010). doi: 10.3141/2147-09

38. Ching-Yao Chan (ed.): Defining Safety Performance Measures of Driver-Assistance Systems for Intersection Left-Turn Conflicts. Intelligent Vehicles Symposium, 2006 IEEE. Intelligent Vehicles Symposium, 2006 IEEE (2006)

39. Winner, H., Geyer, S., Sefati, M.: Maße für den Sicherheitsgewinn von Fahrerassistenzsystemen. In: Winner, H., Bruder, R. (Hrsg.) Maßstäbe des sicheren Fahrens. 6. Darmstädter Kolloquium Mensch + Fahrzeug. Ergonomia Verlag, Stuttgart (2013) 
40. Dijkstra, A., Drolenga, H.: Safety effects of route choice in a road network. Simulation of changing route choice. SWOV Institute for Road Safety Research, Leidschendam, Netherlands (2008)

41. Yang, H.: Simulation-based evaluation of traffic safety performance using surrogate safety measures (2012)

42. Zhang, Y., Antonsson, E.K., Grote, K.: A new threat assessment measure for collision avoidance systems. Intelligent Transportation Systems Conference, 2006. ITSC ‘06. IEEE

43. Jansson, J.: Collision avoidance theory with application to automotive collision mitigation. Linköping studies in science and technology. Dissertations, vol. 950. Dept. of Electrical Enginering, Univ., Linköping (2005)

44. Horst, A. R. A. van der: A time-based analysis of road user behaviour in normal and critical encounters. Institute for Perception TNO, Soesterberg, Netherlands (1990)

45. Tamke, A., Dang, T., Breuel, G.: A flexible method for criticality assessment in driver assistance systems. Intelligent Vehicles Symposium (IV), 2011 IEEE

46. Althoff, M., Stursberg, O., Buss, M.: Model-Based Probabilistic Collision Detection in Autonomous Driving. Intelligent Transportation Systems, IEEE Transactions on 10(2), 299-310 (2009). doi: 10.1109/TITS.2009.2018966

47. Althoff, D., Wollherr, D., Buss, M.: Safety assessment of trajectories for navigation in uncertain and dynamic environments. Robotics and Automation (ICRA), 2011 IEEE International Conference on

48. Althoff, D., Kuffner, J., Wollherr, D., Buss, M.: Safety assessment of robot trajectories for navigation in uncertain and dynamic environments. Auton Robot 32(3), 285-302 (2012). doi: 10.1007/s10514-011-9257-9

49. Meier, A., Gonter, M., Kruse, R.: Symbolic Regression for Precrash Accident Severity Prediction. In: Polycarpou, M., Carvalho, A. de, Pan, J.-S., Woźniak, M., Quintian, H., Corchado, E. (Hrsg.) Hybrid Artificial Intelligence Systems, vol. 8480. Lecture Notes in Computer Science, pp. 133-144. Springer International Publishing (2014)

50. Mukherjee, S., Chawla, A., Mohan, D., Singh, M., Dey, R.: Effect of vehicle design on head injury severity and throw distance variations in bicycle crashes. Proceedings From 20th International Technical Conference on the Enhanced Safety of Vehicles. Lyon (2007)

51. Czarnowski, I., Jędrzejowicz, P.: Machine Learning and Multiagent Systems as Interrelated Technologies. In: Czarnowski, I., Jędrzejowicz, P., Kacprzyk, J. (Hrsg.) Agent-Based Optimization, vol. 456. Studies in computational intelligence, pp. 1-28. Springer Berlin Heidelberg (2013)

52. Miller, P.: Die Intelligenz des Schwarms. Was wir von Tieren für unser Leben in einer komplexen Welt lernen können. Campus-Verl., Frankfurt am Main [u.a.] (2010)

53. Gifford, C.M.: Collective Machine Learning: Team Learning and Classification in Multi-agent Systems. Dissertation, University of Kansas (2009) 